\title{
Squeezing and releasing
}

The GTPase dynamin forms helical structures around the necks of budding vesicles and has a key role in membrane fission. Two studies in Cell now show that dynamin assemblies squeeze the lipid bilayers and that GTP hydrolysis triggers disassembly. In the constant presence of GTP, dynamin undergoes multiple rounds of assembly and disassembly, leading to stochastic membrane fission events.

Using real-time microscopy, Pucadyil and Schmid observed that dynamin self-assembles on so-called SUPER templates (lipid bilayers that surround microbeads), which form long membrane tubules. The addition of GTP causes the disassembly of fluorescently labelled dynamin from the tubules, but not membrane fission. However, in the constant presence of GTP (which represents a more physiological condition), dynamin promotes the release of vesicles from SUPER templates. Further, fluctuations in fluorescence intensity of labelled dynamin collars that are assembled at the bud neck suggest that, in the presence of GTP, dynamin assembly is self-limited by cyclic rounds of assembly and disassembly.

Bashkirov et al. used electrophysiology techniques to measure the ionic permeability (conductance) of lipid nanotubules (NTs) that were pulled from membrane bilayers. As NT conductance decreases when NTs become thinner, the authors could monitor dynamin-induced changes of NT diameters in real time. In the absence of GTP, long dynamin assemblies coat the NTs and squeeze them to narrow radii. Upon squeezing, these assemblies stabilize the NT near the point of instability, so that NT constriction by dynamin assemblies does not promote membrane fission. In the constant presence of GTP, however, periodic variations of NT conductance reveal the formation of short and transient dynamin assemblies on the NTs, and membrane fission occurs spontaneously during cycles

of dynamin assembly and disassembly. Thus, a short dynamin scaffold that brings a narrow membrane tubule close to the point of curvature instability is the key intermediate structure in membrane fission. Furthermore, by varying the lipid composition of NTs to change the membrane rigidity, Bashkirov et al. found that dynamin-mediated squeezing and fission is lipid dependent.

Based on a mathematical model, Bashkirov et al. propose that "local narrowing of NT could induce cooperative lipid tilting, leading to self-merger of the inner monolayer of NT (hemifission)". Indeed, simultaneously measuring the NT conductance and the ionic current through the NT membrane did not detect any leakage, thereby suggesting that membrane fission occurs through hemifission. It will be interesting to study whether this mechanism of membrane fission is unique to dynamin or is shared with other GTPases.

Francesca Cesari

ORIGINAL RESEARCH PAPERS Pucadyil, T. J. \& Schmid, S. L. Real-time visualization of dynamin-catalyzed membrane fission and vesicle release. Cell 135, 1263-1275 (2008)| Bashkirov, P. V. et al. GTPase cycle of dynamin is coupled to membrane squeeze and release, leading to spontaneous fission. Cell 135, 1276-1286 (2008)

FURTHER READING Zimmerberg, J. \&

Kozlov, M. M. How proteins produce cellular membrane curvature. Nature Rev. Mol. Cell Biol. 7 , 9-19 (2006) | Martens, S. \& McMahon, H. T. Mechanisms of membrane fusion: disparate players and common principles. Nature Rev. Mol. Cell Biol. 9, 543-556 (2008) 